\title{
Colangiografía endoscópica en anatomía modificada
}

\author{
Enrique Murcio-Pérez* \\ Departamento de Endoscopia Gastrointestinal, Centro Médico Nacional Siglo XXI, Instituto Mexicano del Seguro Social, Ciudad de México, México
}

\section{Resumen}

Se presentan los trabajos de interés relacionados a la colangiografía retrógrada endoscópica (CRE) en pacientes con anatomía modificada aceptados para su presentación en la Semana de Enfermedades Digestivas (DDW) 2020 a llevarse a cabo en la ciudad de Chicago, IL. USA incluyendo estudios relacionados a la CRE mediante enteroscopia así como varias nuevas técnicas y herramientas relacionadas al acceso biliar o enteral asistido por ultrasonido endoscópico en pacientes con bypass gástrico o anatomía modificada.

Palabras clave: Colangiografía endoscópica retrógrada (CRE). Bypass gástrico. Anatomía modificada.

\section{CRE asistida por laparoscopia posterior a bypass gástrico}

Un estudio retrospectivo de 12 años en un solo centro norteamericano buscó evaluar los desenlaces y curva de aprendizaje en pacientes con bypass gástrico que requirieron CRE asistida por laparoscopia. Se incluyeron 131 pacientes realizando acceso quirúrgico a través del remanente gástrico para lograr introducción del duodenoscopio. La indicación principal fue coledocolitiasis $(78 \%)$ y la mediana de tiempo entre la realización del bypass gástrico y la CRE fue de 81 meses. El éxito técnico fue de $100 \%$ con una mediana de tiempo para la fase quirúrgica de 128 minutos y una mediana de tiempo de 48 minutos para la fase endoscópica. Ocurrieron complicaciones en el $6 \%$ de los pacientes, siendo la más común infección del sitio de entrada en el remanente gástrico. La tasa de pancreatitis post CRE fue de $3.8 \%$. Fue necesario la reconversión a cirugía abierta en $10.6 \%$ de los casos encontrando como predictores de conversión a cirugía abierta: historia de múltiples intervenciones abdominales (OR 10.4). Se calculó una curva de aprendizaje sumatoria acumulada (CUSUM) mostrando 27 casos para el equipo quirúrgico y 10 casos para el equipo endoscópico para obtener resultados óptimos ${ }^{1}$.

Este trabajo muestra que la CRE asistida por cirugía es efectiva, incluso la gran mayoría pudo ser asistida por laparoscopia. A pesar de haber sido realizada en un solo centro, las herramientas utilizadas se encuentran al alcance de la mayoría de los hospitales que tratan patologías de la vía biliar. Cabe llamar la atención sobre la infección de herida quirúrgica en acceso gástrico, y no se especifican en el artículo las precauciones pre, trans y posprocedimiento, que sería interesante evaluar para tomar estrategias dirigidas a disminuir dicha complicación.

\section{CRE en pacientes ancianos con anatomía modificada}

Un estudio retrospectivo de un solo centro en China buscó evaluar seguridad y factibilidad de la CRE en pacientes mayores de 70 años con anatomía modificada 
por diferentes procedimientos quirúrgicos (Billroth I, Billroth II, anastomosis en Y de Roux y colédoco-duodenostomía). Se incluyeron 36 pacientes. La mediana de edad de los pacientes fue 78 años y la mayoría fueron hombres (75\%). La localización de la obstrucción fue biliar extrahepática en todos los casos. El procedimiento quirúrgico más frecuente de los pacientes fue Billroth II (47\%) seguido de anastomosis en Y de Roux (41\%) y finalmente Billroth I y colédoco-duodenostomía (5.6\% cada uno). Anatómicamente fue encontrado divertículo periampular en el $22 \%$ de los casos. La falla técnica (canulación o extracción fallida de lito) ocurrió en el $36 \%$ de los casos con una tasa de complicaciones del $19.4 \%$ (pancreatitis, colangitis, perforación, hemorragia). Este estudió comparó los desenlaces observados con una cohorte de pacientes $<70$ años encontrando diferencia estadísticamente significativa de mayor comorbilidad y falla técnica en los sujetos $>70$ años $^{2}$.

El presente estudio tiene varias debilidades propias de su diseño. Además de haber sido realizado en un solo centro, no mencionan el endoscopio utilizado para lograr el acceso biliar (enteroscopio, duodenoscopio), el material empleado, ni la experiencia de los endoscopistas. La falla técnica y tasa de complicaciones fueron más altas de lo esperado no especificando si esto generó una mayor estancia hospitalaria o incluso muerte en un grupo de pacientes con mayor comorbilidad. Los datos observados en ese centro invitan a replantearse si la estrategia endoscópica es la adecuada en pacientes mayores de 70 años con litos biliares y anatomía modificada.

\section{CRE gastro-gástrica dirigida por Ultrasonido Endoscópico (EDGE)}

El acceso endoscópico a la vía biliar nativa excluida quirúrgicamente en procedimiento derivativos digestivos no siempre es exitoso. La endoscopia convencional está acostumbrada a utilizar los trayectos propios del tubo digestivo. Sin embargo, el desarrollo de herramientas específicas para crear comunicación entre dos cavidades ha permitido el procedimiento de CRE mediante la creación de un acceso por USE, conocido por el acrónimo de EDGE. Clásicamente, EDGE es un procedimiento en dos pasos. Una primera fase donde se crea la comunicación entre dos estructuras digestiva mediante prótesis metálica de yuxtaposición luminal (LAMS) y un segundo paso semanas después para permitir que la fístula creada madure y permita la realización de la $\mathrm{CRE}^{3}$.

En ocasiones es necesario derivar la vía biliar de forma urgente y se ha realizado EGDE en un solo tiempo con creación de fístula con LAMS seguida de CRE inmediata. Un estudio multicéntrico norteamericano de tipo retrospectivo mostró sus resultados en la realización de EDGE desde la primera sesión. Incluyeron 131 pacientes con una mediana de edad de 58 años. La migración de LAMS durante EDGE ocurrió en el $9 \%$ de los casos. Se analizaron factores predictivos de dicha migración encontrando que un LAMS de $15 \mathrm{~mm}$ tenía mayor riesgo de migración (OR 7.9; IC 95\%: 1.347) en comparación con LAMS de $20 \mathrm{~mm}$. Así mismo el uso de sutura para fijación endoscópica y la dilatación del LAMS posterior a la liberación fueron maniobras asociadas a una menor migración de LAMS ${ }^{4}$.

Otra variante de acceso digestivo dirigido por USE es la anastomosis entero-enteral para lograr la instrumentación de una hepato-yeyuno anastomosis en pacientes con hepato-yeyuno anastomosis en $Y$ de Roux. Un estudio multicéntrico europeo evaluó la factibilidad y desenlaces clínicos del acceso enteral (yeyuno-yeyuno) guiado por USE (AE-USE) en 44 pacientes con bypass en $Y$ de Roux y acceso endoscópico convencional fallido. El éxito técnico del AE-USE fue del 97.7\% Los accesos fueron gastro-yeyunal (62.8\%), duodeno-yeyunal (30.2\%) y yeyuno-yeyunal (6.9\%). El AE-USE fue realizado en un mismo procedimiento en el $23 \%$ de los pacientes. La mediana de días entre AE-USE y la CRE trans yeyunal fue de 7 días. Ocurrieron eventos adversos en el 4 de los casos (2 perforaciones y 2 LAMS con liberación fallida). El éxito en lograr drenaje biliar a través del AE-USE fue del $97.3 \%$ con un éxito en el largo plazo del $70 \% 5$.

De forma similar, un centro italiano presentó su experiencia en el AE-USE en 40 pacientes con estenosis de la hepato-yeyuno anastomosis. El éxito técnico para la creación del acceso enteral dirigido por USE fue del $95 \%$, ocurriendo una perforación y una mala colocación del LAMS. La CRE pudo ser realizada con éxito en el $100 \%$ de los casos donde se logró crear la comunicación yeyunal. El seguimiento clínico tuvo una mediana de 29 meses logrando resolución endoscópica de la estenosis en el $100 \%$ de los casos $^{6}$.

Finalmente, un estudio retrospectivo en un solo centro mostró sus resultados comparando indicaciones, técnicas y resultados de la enteroscopia doble balón (EDB) contra aquellos obtenidos por CRE transgástrica asistida por USE (EDGE) en paciente con bypass gástrico en Y de Roux y patología biliopancreática. Se incluyeron 41 pacientes con una mediana de edad de 65 años realizando 72 procedimientos correspondiendo 32 sujetos al grupo EDB y 9 sujetos a EDGE. El éxito terapéutico fue superior en el grupo EDGE en comparación con EDB ( $100 \%$ vs. $84 \%, p=0.066$ ) aunque no 
fue significativamente estadístico. El tiempo de procedimiento fue menor para EDGE vs. EDB (49.18 minutos vs. $67.2 \mathrm{~min} . \mathrm{P}=0.045$ ) Los efectos adversos fueron más frecuentes en EDGE vs. EDB ( $11 \%$ vs. $6 \% \mathrm{p}=\mathrm{NS})^{7}$.

Los trabajos previos reflejan un avance de la endoscopia fuera del lumen nativo digestivo, situación que se asemeja a la revolución reciente en la endoscopía del tercer espacio. Es claro que requieren de centros con experiencia e instrumentos dedicados para lograr éxito en el AE-USE mismos que por el momento tienen un costo elevado y no son del acceso de todos los centros, aunque podrían considerarse en pacientes que requirieran intervenciones no posibles con enteroscopia (ej., litotricia intraductal). Otro punto para tener en cuenta son las complicaciones, que pueden ser graves y requerir cirugía.

\section{Financiamiento}

Los autores no recibieron patrocinio para llevar a cabo este artículo.

\section{Conflicto de intereses}

Consultor para Boston Scientific México

\section{Bibliografía}

1. Al Masri S, Zenati M, Papachristou G. et al. Laparoscopic assisted endoscopic retrograde cholangiopancreatography following gastric bypass: a 12-year assessment of outcomes and learning curve at a high volumen pancreatobiliary center. DDW: 2020 mayo 2-5; Chicago, IL.893-2020.

2. Zhao S, Miao L, Ji Guozhong. et al. ERCP in the removal of biliopancreatic stones for elderly patients with surgically altered anatomy. DDW: 2020 mayo 2-5; Chicago, IL. Su1452.

3. Kedia P, Sharaiha RZ, Kumta NA, Internal EUS-directed transgastric ERCP (EDGE): game over. Gastroenterology. 2014;147(3):566-568.

4. Shinn B, Boortalary T., Raijman I. et al. Maximizing success in single-session EDGE. Predictive factors for stent migration. DDW: 2020 mayo 2-5; Chicago, IL. Su 1444.

5. Carbajo-López AY, Kunda R., De Benito M. et al. Transjejunal ERCP (TJ-ERCP) via EUS-Guided entero-anastomoses (EUS-ES) in Roux-en-Y-Hepaticojejunostomy: Short and long-term outcomes of a variant EUS-Directed ERCP in a challenging patient population. DDW: 2020 mayo 2-5; Chicago, IL. 958-2020.

6. Mutignani M., Forti E., Pugliese F. et al. Hepático-jejunostomy's anastomotic stricture treated by EUS-guided enteral-enterl endoscopic bypass (EEEB) and subsequent ERC: a tertiary referral single center experience. DDW: 2020 mayo 2-5; Chicago, IL. Su1512

7. Abidali H., Rezaie A., Omer A., et al. EUS-directed transgastric ERCP versus Double Balloon Enteroscopy-Assisted ERCP in patients with rouxen-y gastric bypass anatomy: a single center experience. DDW: 2020 mayo 2-5; Chicago, IL. Su1427. 